\title{
Proposal of the genus Sphingomonas sensu stricto and three new genera, Sphingobium, Novosphingobium and Sphingopyxis, on the basis of phylogenetic and chemotaxonomic analyses
}

\footnotetext{
1 Institute for Fermentation Osaka, 17-85, Jusohonmachi 2-chome, Yodogawa-ku, Osaka 532-8686, Japan

2 Department of Laboratory Sciences, Gunma University School of Health Sciences, 39-15 Showa-machi 3chome, Maebashi, Gunma 371-8514, Japan

3 Department of Ecological Engineering, Toyohashi University of Technology, Tenpaku-cho, Toyohashi 441-8580, Japan
}

\author{
Mariko Takeuchi, ${ }^{1}$ Koei Hamana ${ }^{2}$ and Akira Hiraishi ${ }^{3}$ \\ Author for correspondence: Mariko Takeuchi. Tel: +8166300 6555. Fax: +81663006814. \\ e-mail: takeuchi-mariko@ifo.or.jp
}

\begin{abstract}
Phylogenetic analyses of 165 rRNA gene sequences by distance matrix and parsimony methods indicated that the currently known species of the genus Sphingomonas can be divided into four clusters. Some chemotaxonomic and phenotypic differences were noted among these clusters. Three new genera, Sphingobium, Novosphingobium and Sphingopyxis, are proposed in addition to the genus Sphingomonas sensu stricto. The genus Sphingobium is proposed to accommodate Sphingomonas chlorophenolica, Sphingomonas herbicidovorans and Sphingomonas yanoikuyae. The genus Novosphingobium is proposed for Sphingomonas aromaticivorans, Sphingomonas capsulata, Sphingomonas rosa, Sphingomonas stygia, Sphingomonas subarctica and Sphingomonas subterranea. Sphingomonas macrogoltabidus and Sphingomonas terrae are reclassified in the genus Sphingopyxis. The type species of Sphingobium, Novosphingobium and Sphingopyxis are Sphingobium yanoikuyae, Novosphingobium capsulatum and Sphingopyxis macrogoltabida, respectively.
\end{abstract}

Keywords: Sphingomonas sensu stricto, Sphingobium, Novosphingobium, Sphingopyxis

\section{INTRODUCTION}

The genus Sphingomonas was created by Yabuuchi et al. (1990) to accommodate strictly aerobic, chemoheterotrophic, yellow-pigmented, Gram-negative, rodshaped bacteria that contain glycosphingolipids (GSLs) as cell envelope components. The species of the genus described were Sphingomonas adhaesiva, Sphingomonas capsulata, Sphingomonas parapaucimobilis, Sphingomonas paucimobilis (type species) and Sphingomonas yanoikuyae, most of which consisted of strains of clinical origin. Since then, large numbers of phenotypically and phylogenetically similar strains have been isolated from various environments and

Abbreviations: BChl, bacteriochlorophyll; GSLs, glycosphingolipids.

The DDBJ accession numbers for the $16 \mathrm{~S}$ rDNA sequences of Sphingomonas spp. IFO 15914, IFO 15496, IFO 15497, IFO 15495, IFO 15915 and IFO 15917 and Sphingomonas herbicidovorum IFO $16415^{\top}$ are AB033945-AB033950 and $\mathrm{AB} 042233$, respectively. described as novel species of this genus. These include Sphingomonas macrogoltabidus, Sphingomonas sanguinis, Sphingomonas terrae (Takeuchi et al., 1993), Sphingomonas asaccharolytica, Sphingomonas mali, Sphingomonas pruni, Sphingomonas rosa (Takeuchi et al., 1995), Sphingomonas chlorophenolica (Nohynek et al., 1995), Sphingomonas herbicidovorans (Zipper et al., 1996), Sphingomonas subarctica (Nohynek et al., 1996), Sphingomonas aromaticivorans, Sphingomonas stygia, Sphingomonas subterranea (Balkwill et al., 1997), Sphingomonas trueperi (Kämpfer et al., 1997), 'Sphingomonas agrestis' strain HV3 (Yrjälä et al., 1998) and Sphingomonas echinoides (Denner et al., 1999). Yabuuchi et al. (1999) transferred Blastomonas natatoria (Sly \& Cahill, 1997), Erythromonas ursincola (Yurkov et al., 1997) and Rhizomonas suberifaciens (Van Bruggen et al., 1990, 1993) to the genus Sphingomonas as Sphingomonas natatoria, Sphingomonas ursincola and Sphingomonas suberifaciens, respectively. Thus, the increase in the number of species in the genus Sphingomonas has complicated Sphingo- 
monas classification. The proposal of Yabuuchi et al. (1999) would place a broader range of species, including photosynthetic and phytopathogenic species, in the genus Sphingomonas.

In contrast, Hiraishi et al. (2000) emended the description of Blastomonas as a genus of aerobic photosynthetic bacteria and reclassified Erythromonas (Sphingomonas) ursincola as a species of the genus Blastomonas. Segers et al. (1994) suggested that S. yanoikuyae and $S$. capsulata should belong to a genus separate from the genus Sphingomonas; this suggestion was supported by polyamine profiling (Nohynek et al., 1996; Denner et al., 1999). In addition, Kämpfer et al. (1997) indicated that the Sphingomonas species containing sym-homospermidine as the major polyamine, i.e. S. paucimobilis, S. mali, S. pruni and S. trueperi, formed a relatively homogeneous subgroup within the genus.

The genus now contains genetically relatively diverse species that can be classified into at least four clusters within the $\alpha-4$ subclass of the Proteobacteria based on 16S rDNA sequences (Stackebrandt et al., 1988; Takeuchi et al., 1994; Yurkov et al., 1997). The chemotaxonomic and phylogenetic heterogeneity existing in the genus Sphingomonas calls for further study regarding this genus and related sphingomonads.

In this study, almost-complete 16S rRNA gene sequences of several strains of the genus Sphingomonas and related genera were examined and sequences were compared with published sequences of species belonging to the $\alpha-4$ subclass of the Proteobacteria. Fatty acid profiles, polyamine patterns and some other traits of the sphingomonads were also examined. Based on the results of these phylogenetic, chemotaxonomic and phenotypic approaches, the transfer of several species from the genus Sphingomonas to three new genera, Sphingobium, Novosphingobium and Sphingopyxis, is proposed. The description of the genus Sphingomonas is also emended.

\section{METHODS}

Bacterial strains and cultivation. The strains used in this study are listed in Table 1 . All of the test strains were cultured aerobically at $30{ }^{\circ} \mathrm{C}$ in PY medium, which contained $\left(1^{-1}\right.$ distilled water $): 10 \mathrm{~g}$ peptone, $2 \mathrm{~g}$ yeast extract and $1 \mathrm{~g} \mathrm{MgSO}_{4} \cdot 7 \mathrm{H}_{2} \mathrm{O}(\mathrm{pH} \mathrm{7 \cdot 0)}$. For fatty acid analysis of sphingolipids, all strains were also cultured in R (Rich) medium. $\mathrm{R}$ medium contained $\left(\mathrm{l}^{-1}\right.$ distilled water $): 10 \mathrm{~g}$ peptone, $5 \mathrm{~g}$ yeast extract, $5 \mathrm{~g}$ malt extract, $5 \mathrm{~g}$ BactoCasamino acids, $2 \mathrm{~g}$ beef extract, $2 \mathrm{~g}$ glycerol, $50 \mathrm{mg}$ Tween 80 and $1 \mathrm{~g} \mathrm{MgSO}_{4} \cdot 7 \mathrm{H}_{2} \mathrm{O}(\mathrm{pH} 7 \cdot 0)$. For the analysis of polyamines, organisms other than Sphingomonas rosa IFO $15208^{\mathrm{T}}$ were cultured aerobically at $30^{\circ} \mathrm{C}$ with shaking in PY medium. S. rosa IFO $15208^{\mathrm{T}}$ was cultured at $30^{\circ} \mathrm{C}$ in PTYG medium containing ( $1^{-1}$ distilled water): $0.25 \mathrm{~g}$ peptone, $0.25 \mathrm{~g}$ tryptone, $0.5 \mathrm{~g}$ yeast extract, $0.5 \mathrm{~g}$ glucose, $0.6 \mathrm{~g}$ $\mathrm{MgSO}_{4} \cdot 7 \mathrm{H}_{2} \mathrm{O}$ and $70 \mathrm{mg} \mathrm{CaCl} 2 \cdot 2 \mathrm{H}_{2} \mathrm{O}(\mathrm{pH} 7 \cdot 0)$.

Cellular fatty acid analysis. Cells were harvested from cultures at the mid-exponential phase of growth and freezedried. Cellular fatty acids were extracted from dried cells $(50 \mathrm{mg})$ by acid methanolysis, purified (Yokota et al., 1993) and analysed by GLC-MS with a Shimadzu GCMSQP5000 spectrometer combined with a CLASS-5000 MS Workstation computer system. GLC was performed with a Shimadzu GC-17A GC equipped with a BPX70 capillary column (SGE) containing $70 \%$ cyanopropyl equivalent modified siloxane $(50 \mathrm{~m} \times 0.25 \mathrm{~mm})$. Components were separated with helium as carrier gas at a flow rate of $1.4 \mathrm{ml} \mathrm{min}{ }^{-1}$ under the following thermal conditions: $80^{\circ} \mathrm{C}$ for $2 \mathrm{~min}, 80-150{ }^{\circ} \mathrm{C}$ at $15^{\circ} \mathrm{C} \min ^{-1}, 150-250{ }^{\circ} \mathrm{C}$ at $8{ }^{\circ} \mathrm{C}$ $\min ^{-1}$ and $250^{\circ} \mathrm{C}$ for $5 \mathrm{~min}$.

Glycosphingolipid (GSL) and quinone analyses. GSLs were extracted from dried cells $(50 \mathrm{mg})$ with chloroform/ methanol $(1: 3, \mathrm{v} / \mathrm{v})$ and treated with $0 \cdot 1 \mathrm{M} \mathrm{NaOH}$ at $100^{\circ} \mathrm{C}$ for $1 \mathrm{~h}$. The lipids were separated by TLC with Merck Kieselgel $60 \mathrm{~F}_{254}$ plates using chloroform/methanol/acetic acid/water (100:20:12:5 by vol.) as the developing solvent (Kawahara et al., 1991). The major spot, corresponding to the monosaccharide-type GSL, was scraped from the plate and subjected to fatty acid analysis as noted above.

Analysis of isoprenoid quinones was performed as described previously (Takeuchi et al., 1993).

Polyamine analysis (Hamana \& Takeuchi, 1998). Cells were homogenized in $0.5 \mathrm{M}$ perchloric acid $\left(\mathrm{HClO}_{4}\right)$ and centrifuged. Polyamines in the resultant supernatant were analysed by HPLC on an L-6000 high-speed liquid chromatograph (Hitachi) as described previously (Hamana et al., 1994)

Biochemical tests and photosynthetic properties. Biochemical tests were performed using API 20NE systems according to the manufacturer's instructions (API bioMérieux).

Bacteriochlorophyll (BChl $a$ ) was extracted with acetone/ methanol $(7: 2, \mathrm{v} / \mathrm{v})$ from fresh wet cells and analysed by reverse-phase HPLC as described previously (Hiraishi et al., 1998). The puf genes, encoding the photosynthetic reaction centre and core light-harvesting complex of purple bacteria, were detected by PCR as described by Hiraishi et al. (1998).

$16 \mathrm{~S}$ rDNA sequence determination and phylogenetic analysis. $16 \mathrm{~S}$ rDNA fragments corresponding to positions 8-1540 in the Escherichia coli numbering system (Brosius et al., 1978) were amplified by PCR, sequenced directly with a Thermo Sequenase cycle sequencing kit with 7-deaza-dGTP (Amersham Life Science) and analysed with a LI-COR 4200 (two-dye system) DNA sequencer according to the manufacturer's instructions.

The 16S rDNA sequences were aligned with published sequences available from DDBJ, EMBL and GenBank. Some positions, including gaps and alignment uncertainties, were omitted from the analysis and the final analysis included 1245 bases. Nucleotide substitution rates $\left(K_{\text {nuc }}\right.$ values) (Kimura, 1980) were calculated, a phylogenetic tree was constructed by the neighbour-joining method (Saitou \& Nei, 1987) and the topology of the tree was evaluated by the bootstrap resampling method (Felsenstein, 1985) with 1000 replicates with the CLUSTAL w program (Thompson et al., 1994). Maximum-parsimony analysis was performed with PAUP* (version 4.0b4a) (Swofford, 2000). Parsimonious trees were found by 100 replicates of random-sequence addition heuristic searches with tree bisection-reconstruction branch swapping. 
Table 1. Bacterial strains used in this study and $16 \mathrm{~S}$ rDNA sequence accession numbers

Only accession numbers submitted from this study are given. Abbreviations: ATCC, American Type Culture Collection, Manassas, VA, USA; CIP, Collection of the

Institut Pasteur, Paris, France; DSM, Deutsche Sammlung von Mikroorganismen und Zellkulturen GmbH, Braunschweig, Germany; GIFU, Department of Microbiology,

Gifu University School of Medicine, Gifu, Japan; IAM, Institute of Molecular and Cellular Biosciences, University of Tokyo, Tokyo, Japan; IFO, Institute for

Fermentation, Osaka, Japan; JCM, Japan Collection of Microorganisms, Institute of Physical and Chemical Research (RIKEN), Hirosawa, Wako, Japan; LMG,

Laboratorium voor Microbiologie, Universiteit Gent, Gent, Belgium; NCIB, National Collection of Industrial Bacteria, NCIMB, Aberdeen, UK; NCPPB, National

Collection of Plant-pathogenic Bacteria, Harpenden, UK; NCTC, National Collection of Type Cultures, Central Public Health Laboratory Service, London, UK; SMCC,

Subsurface Microbial Culture Collections, Florida State University, FL, USA.

\begin{tabular}{|c|c|c|c|c|}
\hline Species & IFO no. & Other designation(s) & Source & Accession no. \\
\hline Sphingomonas adhaesiva & $15099^{\mathrm{T}}$ & ATCC $51229^{\mathrm{T}}$, DSM $7418^{\mathrm{T}}$, GIFU $11458^{\mathrm{T}}$ & Sterile water & \\
\hline Sphingomonas aromaticivorans & $15084^{\mathrm{T}}$ & CIP $105152^{\mathrm{T}}$, SMCC F199 & Coastal plain sediments & \\
\hline Sphingomonas asaccharolytica & $15499^{\mathrm{T}}$ & ATCC $51839^{\mathrm{T}}$ & Apple tree roots & \\
\hline Sphingomonas capsulata & $12533^{\mathrm{T}}$ & ATCC $14666^{\mathrm{T}}$, DSM $30196^{\mathrm{T}}$, GIFU $11526^{\mathrm{T}}$ & Stocked distilled water & \\
\hline Sphingomonas chlorophenolica & $16172^{\mathrm{T}}$ & ATCC $33790^{\mathrm{T}}, \mathrm{JCM} 10275^{\mathrm{T}}$ & Soil & \\
\hline Sphingomonas echinoides & $15742^{\mathrm{T}}$ & ATCC $14820^{\mathrm{T}}$, DSM $1805^{\mathrm{T}}$, NCIB $9420^{\mathrm{T}}$ & Plate contaminant & \\
\hline Sphingomonas herbicidovorans & $16415^{\mathrm{T}}$ & DSM $11019^{\mathrm{T}}$ & Soil & AB042233 \\
\hline Sphingomonas macrogoltabidus & $15033^{\mathrm{T}}$ & ATCC $51380^{\mathrm{T}}$, DSM $8826^{\mathrm{T}}$ & Soil & \\
\hline Sphingomonas mali & $15500^{\mathrm{T}}$ & ATCC $51840^{\mathrm{T}}$, DSM $10565^{\mathrm{T}}, \mathrm{JCM} 10193^{\mathrm{T}}$ & Apple tree roots & \\
\hline Sphingomonas parapaucimobilis & $15100^{\mathrm{T}}$ & ATCC $51231^{\mathrm{T}}$, DSM $7463^{\mathrm{T}}$, GIFU $11387^{\mathrm{T}}$ & Urine & \\
\hline Sphingomonas paucimobilis & $13935^{\mathrm{T}}$ & ATCC $29837^{\mathrm{T}}$, DSM $1098^{\mathrm{T}}$, NCTC $11030^{\mathrm{T}}$ & Hospital respirator & \\
\hline Sphingomonas pruni & $15498^{\mathrm{T}}$ & JCM $10277^{\mathrm{T}}$ & Prunus persica roots & \\
\hline Sphingomonas rosa & $15208^{\mathrm{T}}$ & NCPPB $2661^{\mathrm{T}}$, IAM $14222^{\mathrm{T}}$ & Rosa sp. roots & \\
\hline Sphingomonas sanguinis & $13937^{\mathrm{T}}$ & $\mathrm{JCM} 7514^{\mathrm{T}}$ & Blood & \\
\hline Sphingomonas stygia & $16085^{\mathrm{T}}$ & ATCC $70280^{\mathrm{T}}$, CIP $10514^{\mathrm{T}}$, SMCC B0712 & Coastal plain sediments & \\
\hline Sphingomonas subarctica & $16058^{\mathrm{T}}$ & DSM $10700^{\mathrm{T}}, \mathrm{JCM} 10398^{\mathrm{T}}$ & Fluidized-bed reactor & \\
\hline Sphingomonas subterranea & $16086^{\mathrm{T}}$ & ATCC $70279^{\mathrm{T}}$, CIP $105153^{\mathrm{T}}$, SMCC B0478 & Coastal plain sediments & \\
\hline Sphingomonas terrae & $15098^{\mathrm{T}}$ & ATCC $51381^{\mathrm{T}}$, CIP $10419^{\mathrm{T}}, \mathrm{JCM} 10195^{\mathrm{T}}$ & Activated sludge & \\
\hline Sphingomonas trueperi & $16157^{\mathrm{T}}$ & ATCC $12417^{\mathrm{T}}$, DSM $7225^{\mathrm{T}}, \mathrm{LMG} 2142^{\mathrm{T}}$ & Soil & \\
\hline Sphingomonas yanoikuyae & $15102^{\mathrm{T}}$ & ATCC $51230^{\mathrm{T}}$, DSM $7462^{\mathrm{T}}$, GIFU $9882^{\mathrm{T}}$ & Clinical specimen & \\
\hline Sphingomonas sp. OGS-47* & 15495 & - & Paddy soil & AB033948 \\
\hline Sphingomonas sp. Y-22* & 15496 & - & Paddy soil & AB033946 \\
\hline Sphingomonas sp. Y-39* & 15497 & - & Paddy soil & AB033947 \\
\hline Sphingomonas sp. Sus-A† & 15914 & - & Corrosion of stainless steel welds & AB033945 \\
\hline Sphingomonas sp. $\mathrm{Cu}-\mathrm{B} \dagger$ & 15915 & - & Underground water & AB033949 \\
\hline Sphingomonas sp. $\mathrm{Cu}-\mathrm{D} \dagger$ & 15917 & - & Underground water & AB033950 \\
\hline Blastomonas natatoria & $15649^{\mathrm{T}}$ & ATCC $35951^{\mathrm{T}}$, DSM $3183^{\mathrm{T}}, \mathrm{JCM} 10396^{\mathrm{T}}$ & Freshwater swimming pool & \\
\hline Erythrobacter longus & $14126^{\mathrm{T}}$ & ATCC $33941^{\mathrm{T}}$, DSM $6997^{\mathrm{T}}, \mathrm{JCM} 6170^{\mathrm{T}}$ & Seaweed (Enteromorpha linza) & \\
\hline Rhizomonas suberifaciens & $15211^{\mathrm{T}}$ & ATCC $49355^{\mathrm{T}}, \mathrm{JCM} 8521^{\mathrm{T}}$ & Corked lettuce root & \\
\hline
\end{tabular}

* Oyaizu-Masuchi \& Komagata (1988).

$\dagger$ Kikuchi et al. (1998). 


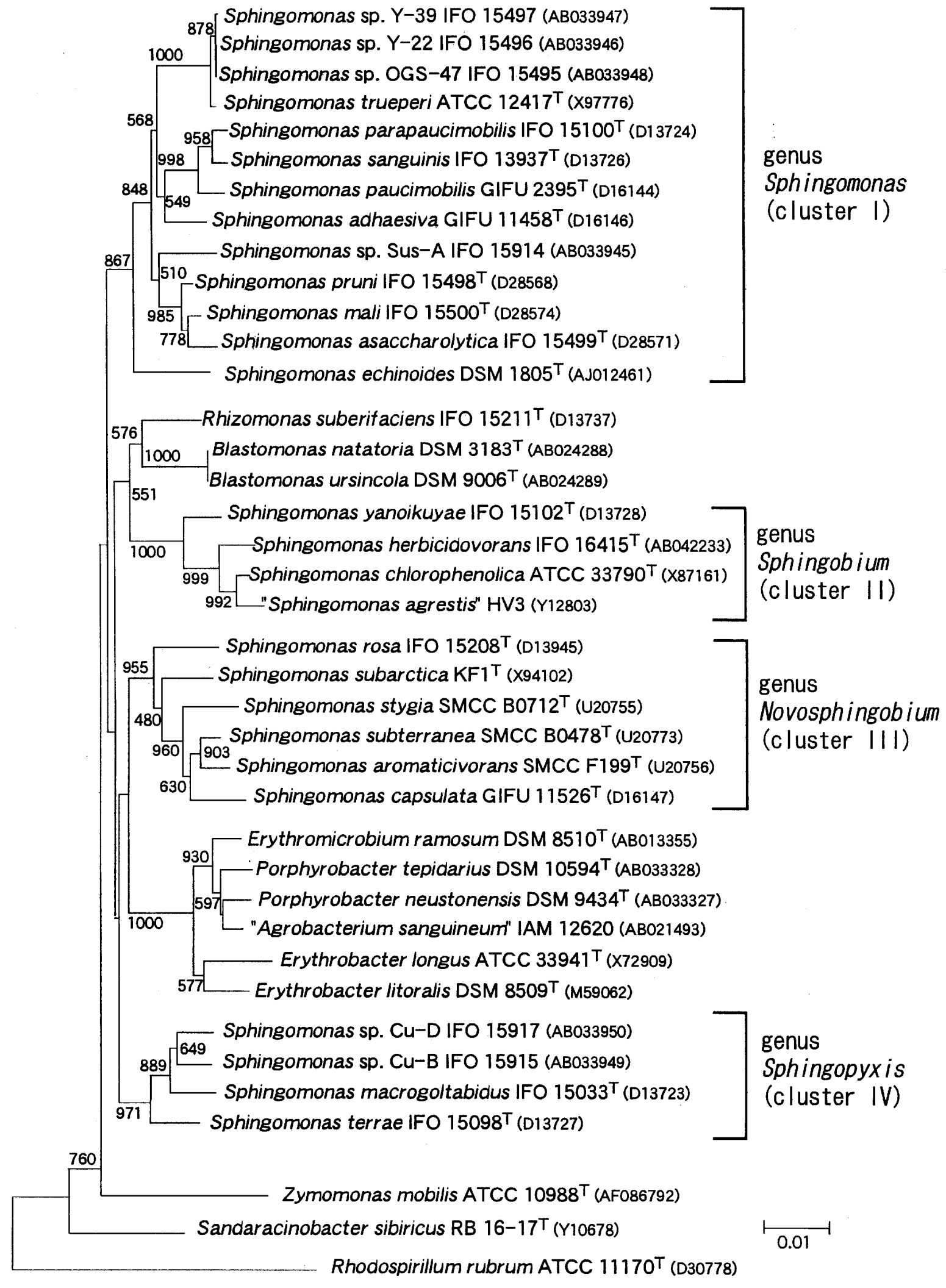

Fig. 1. Phylogenetic tree of Sphingomonas species and their relatives, based on a distance matrix analysis of the $16 \mathrm{~S}$ rDNA sequences (accession numbers given in parentheses). Numbers at the nodes indicate the percentages of bootstrap samplings, derived from 1000 samples, supporting the internal branches (Felsenstein, 1985). Bar, 0.01 $K_{\text {nuc }}$ unit. 


\section{RESULTS}

\section{Phylogenetic analysis}

Almost complete 16S rDNA sequences of the Sphingomonas strains (1395-1468 nt) were determined and compared with the sequences of 30 established and two invalidated species of the genus Sphingomonas and related genera available from the databases. A phylogenetic tree depicting the results of the distance matrix of the $16 \mathrm{~S}$ rDNA sequences shows that the currently known species of Sphingomonas were clearly separated into four clusters, designated here clusters I-IV (Fig. 1). Each of the four clusters could be regarded as a monophyletic and distinct phylogenetic group, as supported by high levels of bootstrap confidence
( $>86 \%)$. The six test strains of unidentified Sphingomonas species were positioned in clusters I and IV. 'Sphingomonas agrestis' strain HV3 (Yrjälä et al., 1998) was positioned in cluster II. The levels of sequence similarity among the Sphingomonas species within each cluster were more than $95.8 \%$, but those between the species of different clusters were lower, ranging from 92.6 to $96.5 \%$ (Table 2). These interrelationships among the four Sphingomonas clusters suggest that each of the clusters corresponds to a single genus from a phylogenetic viewpoint.

Two types of phylogenetic trees were obtained by applying the maximum-parsimony method and both trees also showed that species of the genus Sphingomonas were clearly separated into four clusters (data

Table 2. Levels of $16 \mathrm{~S}$ rDNA sequence similarity among species in the four clusters of Sphingomonas and related micro-organisms

Species: 1, S. paucimobilis; 2, S. yanoikuyae; 3, S. capsulata; 4, S. macrogoltabidus.

\begin{tabular}{|c|c|c|c|c|}
\hline \multirow[t]{2}{*}{ Species and strain } & \multicolumn{4}{|c|}{ Similarity (\%) to species: } \\
\hline & 1 & 2 & 3 & 4 \\
\hline Cluster I (genus Sphingomonas) & $95 \cdot 8-98 \cdot 7$ & $94 \cdot 7-95 \cdot 7$ & $93 \cdot 2-94 \cdot 1$ & $93 \cdot 4-95 \cdot 3$ \\
\hline Cluster II (genus Sphingobium) & $93 \cdot 4-94 \cdot 9$ & $96 \cdot 9-97 \cdot 4$ & $92 \cdot 6-94 \cdot 2$ & $93 \cdot 6-94 \cdot 3$ \\
\hline Cluster III (genus Novosphingobium) & $93 \cdot 2-94 \cdot 3$ & $94 \cdot 2-95 \cdot 1$ & $96 \cdot 7-97 \cdot 8$ & $94 \cdot 8-96 \cdot 5$ \\
\hline Cluster IV (genus Sphingopyxis) & $94 \cdot 1-94 \cdot 9$ & $94 \cdot 7-95 \cdot 7$ & $94 \cdot 1-94 \cdot 8$ & $97 \cdot 6-98 \cdot 0$ \\
\hline Blastomonas natatoria DSM $3183^{\mathrm{T}}$ & $94 \cdot 2$ & $95 \cdot 3$ & $95 \cdot 8$ & $96 \cdot 2$ \\
\hline Blastomonas ursincola DSM $9006^{\mathrm{T}}$ & $94 \cdot 2$ & $95 \cdot 3$ & $95 \cdot 8$ & $96 \cdot 2$ \\
\hline Erythrobacter litoralis DSM $8509^{\mathrm{T}}$ & $93 \cdot 7$ & $93 \cdot 8$ & $94 \cdot 4$ & $94 \cdot 1$ \\
\hline Erythrobacter longus ATCC $33941^{\mathrm{T} *}$ & $92 \cdot 9$ & $93 \cdot 9$ & $94 \cdot 2$ & $93 \cdot 7$ \\
\hline Erythromicrobium ramosum DSM $8510^{\mathrm{T}}$ & $93 \cdot 6$ & $93 \cdot 7$ & $94 \cdot 8$ & $94 \cdot 9$ \\
\hline Porphyrobacter neustonensis DSM $9434^{\mathrm{T}}$ & $93 \cdot 2$ & $93 \cdot 6$ & $94 \cdot 7$ & $94 \cdot 2$ \\
\hline Porphyrobacter tepidarius DSM $10594^{\mathrm{T}}$ & $93 \cdot 3$ & $93 \cdot 4$ & $94 \cdot 5$ & $94 \cdot 2$ \\
\hline Rhizomonas suberifaciens IFO $15211^{\mathrm{T}}$ & $94 \cdot 5$ & $96 \cdot 4$ & $94 \cdot 7$ & $95 \cdot 6$ \\
\hline
\end{tabular}

* Yurkov et al. (1994).

Table 3. Nucleotide signatures of $16 \mathrm{~S}$ rRNA useful for differentiation of the four clusters of Sphingomonas and other sphingolipid-containing genera in the $\alpha-4$ subclass of the Proteobacteria

\begin{tabular}{|c|c|c|c|c|c|c|}
\hline \multirow[t]{2}{*}{ Phylogenetic cluster and genus } & \multirow[t]{2}{*}{ No. of species* } & \multicolumn{5}{|c|}{ Signature at position: } \\
\hline & & $52: 359$ & 134 & 593 & $987: 1218$ & $990: 1215$ \\
\hline Cluster I (genus Sphingomonas) & $9(4)$ & $C: G$ & $\mathrm{G}$ & $\mathrm{G}$ & $\mathrm{G}: \mathrm{C}$ & $\mathrm{U}: \mathrm{G}$ \\
\hline Cluster II (genus Sphingobium) & $4 \dagger$ & $\mathrm{U}: \mathrm{A}$ & G & $\mathrm{U}$ & $A: U$ & $\mathrm{U}: \mathrm{G}$ \\
\hline Cluster III (genus Novosphingobium) & 6 & $\mathrm{C}: \mathrm{G}$ & $\mathrm{G}$ & $\mathrm{U}$ & $\mathrm{G}: \mathrm{C}$ & $\mathrm{U}: \mathrm{A}$ \\
\hline Cluster IV (genus Sphingopyxis) & $2(2)$ & $\mathrm{C}: \mathrm{G}$ & $\mathrm{G}$ & $\mathrm{U}$ & $\mathrm{G}: \mathrm{C}$ & $\mathrm{U}: \mathrm{G}$ \\
\hline Genus Rhizomonas & 1 & $\mathrm{C}: \mathrm{G}$ & A & A & $\mathrm{G}: \mathrm{C}$ & $\mathrm{U}: \mathrm{G}$ \\
\hline Genus Blastomonas & 2 & $C: G$ & $\mathrm{G}$ & $\mathrm{U}$ & $\mathrm{G}: \mathrm{C}$ & $\mathrm{U}: \mathrm{A}$ \\
\hline Genus Erythrobacter & 2 & $C: G$ & $\mathrm{G}$ & $\mathrm{U} / \mathrm{C}$ & $\mathrm{G}: \mathrm{C}$ & $\mathrm{U}: \mathrm{A}$ \\
\hline Genus Porphyrobacter & 2 & $C: G$ & $\mathrm{G}$ & $\mathrm{U}$ & $\mathrm{G}: \mathrm{C}$ & $\mathrm{U}: \mathrm{A}$ \\
\hline
\end{tabular}

* Figures in parentheses indicate the number of test strains of unidentified Sphingomonas species.

$\dagger$ Includes 'Sphingomonas agrestis' strain HV3 (Yrjälä et al., 1998), which has not been validly described. 
Values are percentages of total fatty acid content. -, Not detected.

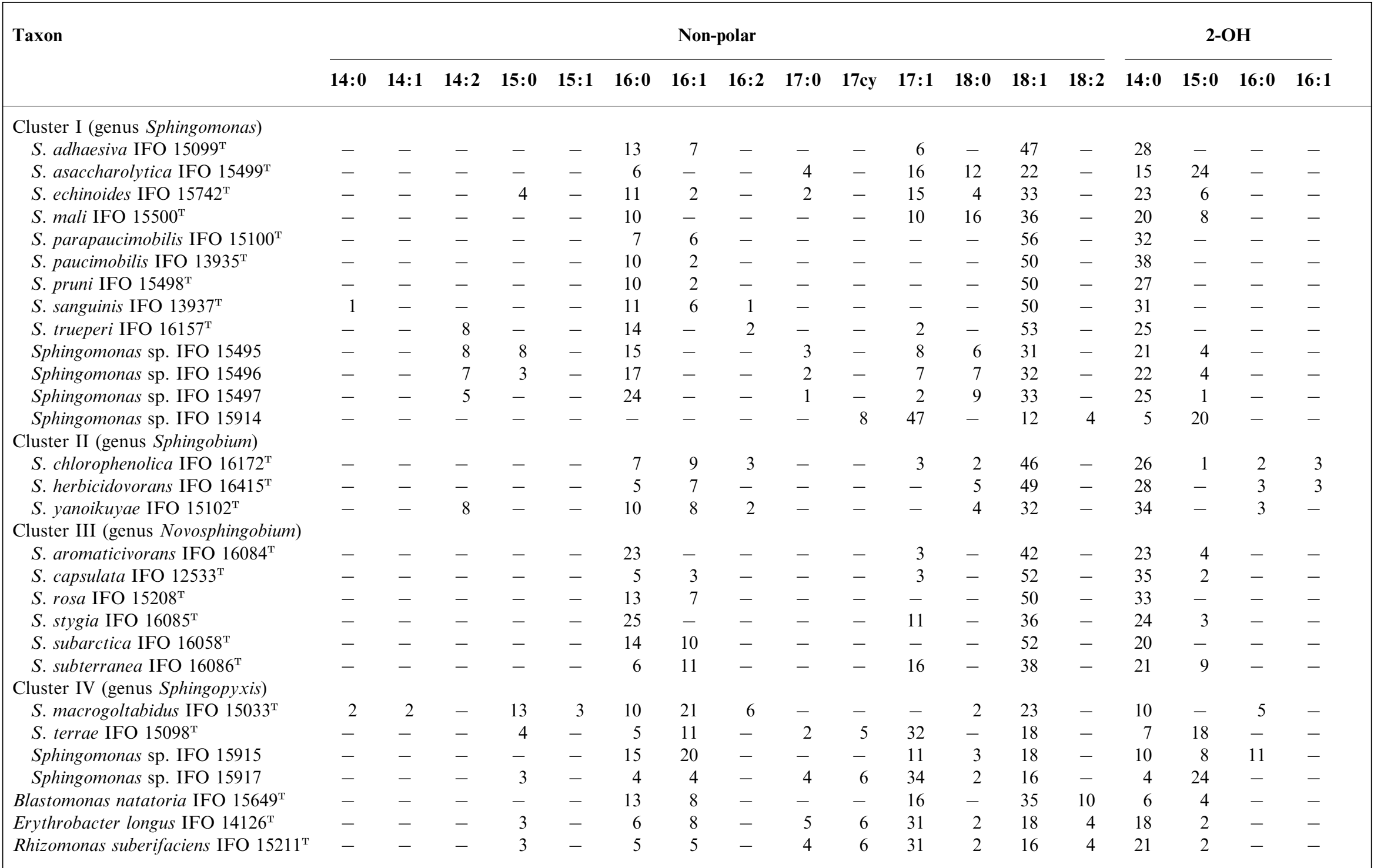


Table 5. 2-Hydroxy fatty acid composition in sphingolipids of species of the genus Sphingomonas and related genera grown in different media

Percentages of total 2-hydroxy fatty acid content in the medium listed are shown.

\begin{tabular}{|c|c|c|c|c|c|c|c|}
\hline \multirow[t]{2}{*}{ Taxon } & \multicolumn{4}{|c|}{ PY medium } & \multicolumn{3}{|c|}{$\mathbf{R}$ medium } \\
\hline & 14:0 & $15: 0$ & $16: 0$ & $16: 1$ & $14: 0$ & $15: 0$ & $16: 0$ \\
\hline \multicolumn{8}{|l|}{ Cluster I (genus Sphingomonas) } \\
\hline S. adhaesiva IFO $15099^{\mathrm{T}}$ & 100 & - & - & - & 100 & - & - \\
\hline S. asaccharolytica IFO $15499^{\mathrm{T}}$ & 39 & 61 & - & - & 18 & 82 & - \\
\hline S. echinoides IFO $15742^{\mathrm{T}}$ & 79 & 21 & - & - & 100 & - & - \\
\hline S. mali IFO $15500^{\mathrm{T}}$ & 71 & 29 & - & - & 75 & 25 & - \\
\hline S. parapaucimobilis IFO $15100^{\mathrm{T}}$ & 100 & - & - & - & 100 & - & - \\
\hline S. paucimobilis IFO $13935^{\mathrm{T}}$ & 100 & - & - & - & 100 & - & - \\
\hline S. pruni IFO $15498^{\mathrm{T}}$ & 86 & 14 & - & - & 100 & - & - \\
\hline S. sanguinis IFO $13937^{\mathrm{T}}$ & 100 & - & - & - & 100 & - & - \\
\hline S. trueperi IFO $16157^{\mathrm{T}}$ & 98 & 2 & - & - & 100 & - & - \\
\hline Sphingomonas sp. IFO 15495 & 81 & 19 & - & - & 100 & - & - \\
\hline Sphingomonas sp. IFO 15496 & 84 & 16 & - & - & 100 & - & - \\
\hline Sphingomonas sp. IFO 15497 & 96 & 4 & - & - & 100 & - & - \\
\hline Sphingomonas sp. IFO 15914 & 18 & 82 & - & - & 25 & 75 & - \\
\hline \multicolumn{8}{|l|}{ Cluster II (genus Sphingobium) } \\
\hline S. chlorophenolica IFO $16172^{\mathrm{T}}$ & 84 & 3 & 5 & 8 & 100 & - & - \\
\hline S. herbicidovorans IFO $16415^{\mathrm{T}}$ & 83 & - & 8 & 9 & 100 & - & - \\
\hline S. yanoikuyae IFO $15102^{\mathrm{T}}$ & 92 & - & 8 & - & 100 & - & - \\
\hline \multicolumn{8}{|l|}{ Cluster III (genus Novosphingobium) } \\
\hline S. aromaticivorans IFO $16084^{\mathrm{T}}$ & 87 & 13 & - & - & 100 & - & - \\
\hline S. capsulata IFO $12533^{\mathrm{T}}$ & 96 & 4 & - & - & 100 & - & - \\
\hline S. rosa IFO $15208^{\mathrm{T}}$ & 100 & - & - & - & 100 & - & - \\
\hline S. stygia IFO $16085^{\mathrm{T}}$ & 89 & 11 & - & - & 100 & - & - \\
\hline S. subarctica IFO $16058^{\mathrm{T}}$ & 87 & - & 13 & - & 100 & - & - \\
\hline S. subterranea IFO $16086^{\mathrm{T}}$ & 70 & 30 & - & - & 100 & - & - \\
\hline \multicolumn{8}{|l|}{ Cluster IV (genus Sphingopyxis) } \\
\hline S. macrogoltabidus IFO $15033^{\mathrm{T}}$ & 67 & - & 33 & - & 62 & - & 38 \\
\hline S. terrae IFO $15098^{\mathrm{T}}$ & 32 & 51 & 12 & 5 & 39 & 52 & 9 \\
\hline Sphingomonas sp. IFO 15915 & 34 & 27 & 28 & 1 & ND & - & - \\
\hline Sphingomonas sp. IFO 15917 & 13 & 75 & 7 & 5 & ND & - & - \\
\hline Blastomonas natatoria IFO $15649^{\mathrm{T}}$ & 35 & 23 & 15 & 27 & 16 & 79 & 5 \\
\hline Blastomonas ursincola DSM $9006^{\mathrm{T}}$ & 60 & 26 & 14 & - & 59 & 22 & 19 \\
\hline Erythrobacter longus IFO $14126^{\mathrm{T}}$ & 13 & 75 & 6 & 6 & 16 & 74 & 10 \\
\hline Porphyrobacter neustonensis DSM $9434^{\mathrm{T}}$ & 54 & 24 & 22 & - & ND & - & - \\
\hline Rhizomonas suberifaciens IFO $15211^{\mathrm{T}}$ & 90 & 10 & - & - & 84 & 16 & - \\
\hline
\end{tabular}

ND, Not determined; - , not detected.

not shown). Each of the phylogenetic trees derived from the neighbour-joining and maximum-parsimony methods showed that the phytopathogenic genus Rhizomonas (Van Bruggen et al., 1990) and the photosynthetic genera Blastomonas (Sly \& Cahill, 1997), Erythrobacter (Shiba \& Simidu, 1982) and Porphyrobacter (Fuerst et al., 1993) were nested in the whole cluster of Sphingomonas species, but were clearly separated from the above-noted four clusters.

Attempts to find nucleotide signatures specific to the 16S rRNA of the four Sphingomonas clusters gave some positive results (Table 3 ). Cluster I was charac- terized by the presence of $\mathrm{G}$ at position 593, cluster II by a U:A pair at position $52: 359$, cluster III by a U:A pair at position 990:1215 and cluster IV by a combination of signatures at these positions. This set of nucleotide signatures may be useful for the circumscription of the four clusters and for their placement in different genera.

\section{Cellular fatty acid profiles}

As shown in Table 4, most of the Sphingomonas strains tested contained 18:1 as the primary component and 16:0 as the second most common type of whole-cell 
Table 6. Cellular concentrations of polyamines in Sphingomonas species and related micro-organisms

Values are $\mu$ mol polyamine (g wet cells) ${ }^{-1}$. Abbreviations: Put, putrescine; Spd, spermidine; HSpd, homospermidine; Agm, agmatine. Data for Blastomonas natatoria and Erythrobacter longus were taken from Hamana \& Takeuchi (1998). -, Not detected. All strains were grown on PY medium with the exception of $S$. rosa IFO $15208^{\mathrm{T}}$, which was grown on PTYG medium.

\begin{tabular}{|c|c|c|c|c|}
\hline Taxon & Put & Spd & HSpd & Agm \\
\hline \multicolumn{5}{|l|}{ Cluster I (genus Sphingomonas) } \\
\hline S. adhaesiva IFO $15099^{\mathrm{T}}$ & - & - & 1.90 & - \\
\hline S. asaccharolytica IFO $15499^{\mathrm{T}}$ & - & - & $1 \cdot 30$ & - \\
\hline S. echinoides IFO $15742^{\mathrm{T}}$ & - & - & $1 \cdot 76$ & - \\
\hline S. mali IFO $15500^{\mathrm{T}}$ & - & - & $0 \cdot 75$ & - \\
\hline S. parapaucimobilis IFO $15100^{\mathrm{T}}$ & - & - & $2 \cdot 35$ & - \\
\hline S. paucimobilis IFO $13953^{\mathrm{T}}$ & - & - & $2 \cdot 55$ & - \\
\hline S. pruni IFO $15498^{\mathrm{T}}$ & - & - & $1 \cdot 42$ & - \\
\hline S. sanguinis IFO $13937^{\mathrm{T}}$ & - & - & 1.97 & - \\
\hline S. trueperi IFO $16157^{\mathrm{T}}$ & - & - & $1 \cdot 10$ & - \\
\hline Sphingomonas sp. IFO 15495 & - & - & $0 \cdot 98$ & - \\
\hline Sphingomonas sp. IFO 15496 & - & - & $1 \cdot 46$ & - \\
\hline Sphingomonas sp. IFO 15497 & - & - & $1 \cdot 20$ & - \\
\hline Sphingomonas sp. IFO 15914 & - & - & $2 \cdot 10$ & - \\
\hline \multicolumn{5}{|l|}{ Cluster II (genus Sphingobium) } \\
\hline S. chlorophenolica IFO $16172^{\mathrm{T}}$ & - & 0.95 & - & - \\
\hline S. herbicidovorans IFO $16415^{\mathrm{T}}$ & - & $1 \cdot 25$ & - & - \\
\hline S. yanoikuyae IFO $15102^{\mathrm{T}}$ & - & $1 \cdot 57$ & - & 0.03 \\
\hline \multicolumn{5}{|l|}{ Cluster III (genus Novosphingobium) } \\
\hline S. aromaticivorans IFO $15084^{T}$ & - & $1 \cdot 05$ & - & - \\
\hline S. capsulata IFO $12533^{\mathrm{T}}$ & $0 \cdot 10$ & $1 \cdot 80$ & - & $0 \cdot 10$ \\
\hline S. rosa $\mathrm{IFO} 15208^{\mathrm{T}}$ & - & $1 \cdot 30$ & - & - \\
\hline S. stygia IFO $16085^{\mathrm{T}}$ & - & 0.95 & - & - \\
\hline S. subarctica IFO $16058^{\mathrm{T}}$ & - & $1 \cdot 00$ & - & - \\
\hline S. subterranea IFO $16086^{\mathrm{T}}$ & - & $1 \cdot 05$ & - & - \\
\hline \multicolumn{5}{|l|}{ Cluster IV (genus Sphingopyxis) } \\
\hline S. macrogoltabidus IFO $15033^{\mathrm{T}}$ & 0.02 & $2 \cdot 10$ & - & - \\
\hline S. terrae IFO $15098^{\mathrm{T}}$ & 0.02 & $2 \cdot 24$ & - & - \\
\hline Sphingomonas sp. IFO 15915 & - & $0 \cdot 85$ & - & $0 \cdot 04$ \\
\hline Blastomonas natatoria IFO $15649^{\mathrm{T}}$ & - & 0.89 & - & 0.01 \\
\hline Erythrobacter longus IFO $14126^{\mathrm{T}}$ & - & $0 \cdot 43$ & - & - \\
\hline Rhizomonas suberifaciens IFO $15211^{\mathrm{T}}$ & 0.01 & 0.58 & - & - \\
\hline
\end{tabular}

fatty acid. Considerable variations were noted in other non-polar fatty acid components among these strains. To determine the usefulness of cellular fatty acid composition, fatty acid profiles of the organisms grown in different media and at different temperatures were examined. However, noticeable differences in cellular fatty acid composition were not found among the test organisms grown under any culture conditions.

\section{GSLs and 2-hydroxy fatty acids}

In TLC experiments, GSLs were detected in all test strains of the genera Sphingomonas, Rhizomonas, Blastomonas, Erythrobacter and Porphyrobacter. The majority of the Sphingomonas species contained not only a monosaccharide-type GSL but also one or more oligosaccharide types of GSL, which were distinguishable from each other in their TLC mobility (data not shown). On the other hand, all test strains of Rhizomonas, Blastomonas, Erythrobacter and Porphyrobacter contained monosaccharide-type GSLs only.

GSL fatty acid profiles of the test organisms changed to some extent depending upon the growth medium (Table 5). The fatty acid patterns of cells grown in $\mathrm{R}$ medium appeared to be simpler and more useful for taxonomic purposes than those obtained with PY medium. Namely, when grown in R medium, strains of Sphingomonas cluster I contained 2-OH 14:0 or 2-OH 15:0 as the major components, those of clusters II and III contained 2-OH 14:0 only and those of cluster IV contained 2-OH 14:0, 2-OH 15:0 and 2-OH 16:0. 
Table 7. Some phenotypic characteristics of Sphingomonas species and related genera

Characteristics are scored as: + , positive; $(+)$, weakly positive; - , negative; ND, not determined.

\begin{tabular}{|c|c|c|c|c|}
\hline Taxon & Nitrate reduction & Aesculin & $\beta$-Galactosidase & BChl $a$ production \\
\hline \multicolumn{5}{|l|}{ Cluster I (genus Sphingomonas) } \\
\hline S. adhaesiva IFO $15099^{\mathrm{T}}$ & - & + & + & - \\
\hline S. asaccharolytica IFO $15499^{\mathrm{T}}$ & + & + & + & - \\
\hline S. echinoides IFO $15742^{\mathrm{T}}$ & + & $(+)$ & - & - \\
\hline S. mali IFO $15500^{\mathrm{T}}$ & + & + & + & - \\
\hline S. parapaucimobilis IFO $15100^{\mathrm{T}}$ & $(+)$ & + & + & - \\
\hline S. paucimobilis IFO $13953^{\mathrm{T}}$ & + & + & + & - \\
\hline S. pruni IFO $15498^{\mathrm{T}}$ & + & + & + & - \\
\hline S. sanguinis $\mathrm{IFO} 13937^{\mathrm{T}}$ & + & + & + & - \\
\hline S. trueperi IFO $16157^{\mathrm{T}}$ & + & + & + & - \\
\hline Sphingomonas sp. IFO 15495 & + & + & + & $\mathrm{ND}$ \\
\hline Sphingomonas sp. IFO 15496 & $(+)$ & + & + & $\mathrm{ND}$ \\
\hline Sphingomonas sp. IFO 15497 & + & + & + & $\mathrm{ND}$ \\
\hline Sphingomonas sp. IFO 15914 & - & + & - & $\mathrm{ND}$ \\
\hline \multicolumn{5}{|l|}{ Cluster II (genus Sphingobium) } \\
\hline S. chlorophenolica IFO $16172^{\mathrm{T}}$ & - & + & + & - \\
\hline S. herbicidovorans IFO $16415^{\mathrm{T}}$ & - & + & + & - \\
\hline S. yanoikuyae IFO $15102^{\mathrm{T}}$ & - & + & + & - \\
\hline \multicolumn{5}{|l|}{ Cluster III (genus Novosphingobium) } \\
\hline S. aromaticivorans IFO $15084^{\mathrm{T}}$ & + & + & + & - \\
\hline S. capsulata IFO $12533^{\mathrm{T}}$ & + & $(+)$ & + & - \\
\hline S. rosa IFO $15208^{\mathrm{T}}$ & + & + & + & - \\
\hline S. stygia IFO $16085^{\mathrm{T}}$ & + & + & $(+)$ & - \\
\hline S. subarctica IFO $16058^{\mathrm{T}}$ & + & + & + & - \\
\hline S. subterranea IFO $16086^{\mathrm{T}}$ & + & + & + & - \\
\hline \multicolumn{5}{|l|}{ Cluster IV (genus Sphingopyxis) } \\
\hline S. macrogoltabidus IFO $15033^{\mathrm{T}}$ & - & + & + & - \\
\hline S. terrae IFO $15098^{\mathrm{T}}$ & - & - & - & - \\
\hline Sphingomonas sp. IFO 15915 & - & + & - & ND \\
\hline Sphingomonas sp. IFO 15917 & - & + & $(+)$ & $\mathrm{ND}$ \\
\hline Blastomonas natatoria IFO $15649^{\mathrm{T}}$ & - & + & - & + \\
\hline Blastomonas ursincola DSM $9006^{\mathrm{T}}$ & - & - & - & + \\
\hline Ervthrobacter longus IFO $14126^{\mathrm{T}}$ & - & + & - & + \\
\hline Rhizomonas suberifaciens IFO $15211^{\mathrm{T}}$ & - & + & - & - \\
\hline
\end{tabular}

Rhizomonas suberifaciens contained 2-OH 14:0 and 2$\mathrm{OH}$ 15:0 and the Blastomonas, Erythromonas and Porphyrobacter species contained 2-OH 14:0, 2-OH 15:0 and 2-OH 16:0. These results indicate that, when the culture conditions are standardized, fatty acid analysis provides more reliable information for determining the taxonomy of sphingomonads.

\section{Polyamine patterns}

As shown in Table 6, homospermidine was detected as the major polyamine only in species belonging to cluster I. All other strains examined contained spermidine as the major polyamine. In a previous study, Takeuchi et al. (1995) reported that $S$. asaccharolytica had spermidine as the predominant component, but in this study, the major component detected was homospermidine $[1.30 \mu \mathrm{mol}$ (g wet cell) ${ }^{-1}$, as described by Kämpfer et al. (1997).

\section{Other characteristics}

In experiments with the API 20NE system, all test strains exhibited negative reactions for indole production, glucose fermentation, arginine dihydrolase, urease and gelatin hydrolysis. Representative strains of the four Sphingomonas clusters tested assimilated arabinose, D-mannose, maltose, D-mannitol, $N$-acetylglucosamine, adipate, $n$-caproate, citrate, gluconate and DL-malic acid. Some phenotypic differences were observed among the test strains (nitrate reduction, aesculin hydrolysis and $\beta$-galactosidase activity) (Table 7). In particular, it is worth noting that most test strains of Sphingomonas cluster I and all strains of cluster III were positive for nitrate reduction, whereas 
none of the test strains of clusters II and IV reduced nitrate to nitrite. BChl $a$ was detected from the test strains of Blastomonas and Erythrobacter, but was not detected in appreciable amounts from those of Sphingomonas or Rhizomonas.

PCR detection of puf genes from Sphingomonas strains was unsuccessful. In all test strains of Sphingomonas, Rhizomonas, Blastomonas and Erythrobacter, ubiquinone-10 was the major respiratory quinone, accounting for more than $93 \%$ of the total quinone content.

\section{DISCUSSION}

Since the proposal of the genus Sphingomonas by Yabuuchi et al. (1990), large numbers of novel species from various environments have been added to this genus. As a result, the genus Sphingomonas has come to encompass a relatively broad range of species with respect to physiology, genetics and ecology. One of the most characteristic features of the genus Sphingomonas is the presence of GSLs. Recently, Yabuuchi et al. (1999) proposed that Blastomonas natatoria, Erythromonas ursincola and Rhizomonas suberifaciens should be reclassified as members of the genus Sphingomonas in view of the fact that GSLs are present in all these species. However, GSLs are found in species of other genera of the $\alpha-4$ subclass of the Proteobacteria, such as Erythrobacter and Porphyrobacter, as reported here and elsewhere (Fuerst et al., 1993). Although the presence of GSLs is an important chemotaxonomic criterion, the assignment of species to the genus Sphingomonas should not be based on this criterion alone. Blastomonas natatoria, Erythromonas ursincola and Rhizomonas suberifaciens not only form a line of descent distinct from the four clusters of Sphingomonas species (Fig. 1), but also differ from the latter clusters in having photosynthetic or phytopathogenic traits. Hiraishi et al. (2000) have recently proposed to emend the description of Blastomonas as a genus of aerobic photosynthetic bacteria and to reclassify Erythromonas ursincola as a species of the genus Blastomonas. Thus, it is concluded that the genera Blastomonas and Rhizomonas should be retained as they stand, because of the taxonomic importance of photosynthetic and phytopathogenic properties and the phylogenetic evidence for their taxonomic positions. Blastomonas and Rhizomonas can be distinguished from all Sphingomonas species at the generic level by a combined use of chemotaxonomic and phenotypic markers, as discussed below.

As reported in this study and previously (Yurkov et al., 1997), 16S rDNA-based phylogenetic analysis indicated that members of the genus Sphingomonas can be divided into four phylogenetic groups, here designated clusters I-IV. All these clusters were considered to be monophyletic, based on high levels $(86 \%)$ of bootstrap confidence. While the levels of sequence similarity among species within each cluster were more than $95.8 \%$, the similarity values between species of different clusters were lower, ranging from 92.6 to $96.5 \%$. The levels of similarity among the four clusters appear to be low enough to justify different generic assignments. A comparative analysis of the primary and secondary $16 \mathrm{~S}$ rRNA structures has also revealed that the four clusters can be identified on the basis of a set of characteristic nucleotide signatures (Table 3).

Some chemotaxonomic and phenotypic features support the phylogenetic evidence that the four clusters of Sphingomonas represent different genera. Firstly, 2hydroxy fatty acid profiles of GSLs provide valuable information for the differentiation of the four clusters. Members of cluster I contained 2-OH 14:0 and/or 2$\mathrm{OH}$ 15:0 as the major components, whereas those of cluster IV were characterized by the presence of 2-OH 15:0 and/or 2-OH 16:0 in addition to 2-OH 14:0. Members of clusters II and III contained 2-OH 14:0 only as the major component, as shown in Table 5, although minor components were detected when grown in PY medium. Secondly, polyamine patterns are quite useful in distinguishing cluster I from all other clusters: the major polyamine was homospermidine in cluster I and spermidine in all other clusters. Thirdly, the activity of nitrate reduction was a useful character to distinguish the four clusters from each other. Therefore, the four clusters of the genus Sphingomonas can be differentiated clearly by a combination of these chemotaxonomic and phenotypic markers. The other sphingolipid-containing genera in the $\alpha-4$ subclass, Blastomonas, Erythrobacter and Porphyrobacter, are distinguishable from any of the Sphingomonas clusters by a combination of sphingolipid fatty acid composition, polyamine pattern, BChl $a$ production, nitrate reduction and $\beta$-galactosidase activity.

In conclusion, the creation of three new genera, Sphingobium, Novosphingobium and Sphingopyxis, is proposed to accommodate the species of clusters II, III and IV, respectively. According to Principle and Rule 39a of the Bacteriological Code (Lapage et al., 1992), the genus Sphingomonas, type species S. paucimobilis, is retained and emended.

\section{Emended description of the genus Sphingomonas (Yabuuchi et al. 1990) emend. Yabuuchi et al. 1999}

Cells are Gram-negative, non-sporulating rods measuring 0.3-0.8 $\times 1.0-1.9 \mu \mathrm{m}$. Motile or non-motile. Colonies are yellow or off-white. Strictly aerobic and chemo-organotrophic. Catalase-positive. Respiratory quinone is predominantly Q-10. Major fatty acids are 18:1, saturated 16:0 and/or 17:1. Major 2-hydroxy fatty acids are 2-OH 14:0 or 2-OH 15:0. GSLs are present. Homospermidine is the major polyamine component. The DNA G $+\mathrm{C}$ content is $62-68 \mathrm{~mol} \%$. Phylogenetic position is in the $\alpha-4$ subclass of Proteobacteria. Characteristic 16S rRNA signatures are found at positions 52:359 (C:G), $134(\mathrm{G}), 593(\mathrm{G})$, 987:1218 (G:C) and 990:1215 (U:G). Habitat: soil and clinical specimens. Nine species are assigned to 
the emended genus Sphingomonas: Sphingomonas adhaesiva, Sphingomonas asaccharolytica, Sphingomonas echinoides, Sphingomonas mali, Sphingomonas parapaucimobilis, Sphingomonas paucimobilis, Sphingomonas pruni, Sphingomonas sanguinis and Sphingomonas trueperi. Type species: Sphingomonas paucimobilis Yabuuchi et al. 1990.

\section{Description of Sphingobium gen. nov.}

Sphingobium (Sphin.go'bi.um. Gr. adj. Sphingos of sphinx; Gr. n. bios life, living; N.L. fem. n. Sphingobium sphingosine-containing life).

Cells are Gram-negative, non-sporulating rods, measuring $0.3-0.7 \times 1.0-3.5 \mu \mathrm{m}$. Motile or non-motile. Colonies are yellow or whitish-brown. Strictly aerobic and chemo-organotrophic. Catalase-positive. Nitrate is not reduced. Ubiquinone is predominantly Q-10. The dominant fatty acid is $18: 1$ and $16: 0$ is a minor component. Major 2-hydroxy fatty acid is 2-OH 14:0. GSLs are present. Spermidine is the major polyamine component. The DNA G $+\mathrm{C}$ content is $62-67 \mathrm{~mol} \%$. Characteristic 16S rRNA signatures are present at positions 52:359 (U:A), $134(\mathrm{G}), 593(\mathrm{U}), 987$ : 1218 (A:U) and 990:1215 (U:G). Habitat: soil, pentachlorophenol-contaminated soil and clinical specimens. The type species is Sphingobium yanoikuyae (Yabuuchi et al. 1990) Takeuchi et al. 2001.

\section{Description of Sphingobium chlorophenolicum comb. nov.}

With the addition of the details listed in the genus description above, the description is the same as that given by Nohynek et al. (1995).

\section{Description of Sphingobium herbicidovorans comb. nov.}

With the addition of the details listed in the genus description above, the description is the same as that given by Zipper et al. (1996).

\section{Description of Sphingobium yanoikuyae comb. nov.}

With the addition of the details listed in the genus description above, the description is the same as that given by Yabuuchi et al. (1990).

\section{Description of Novosphingobium gen. nov.}

Novosphingobium (No.vo.sphin.go'bi.um. L. adj. novus new; Gr. adj. sphingos of sphinx; Gr. n. bios life, living; N.L. fem. n. Novosphingobium new sphingosine-containing life).

Cells are Gram-negative, non-sporulating rods measuring $0.3-0.5 \times 1 \cdot 0-3.0 \mu \mathrm{m}$. Motile or non-motile. Colonies are yellow or whitish-brown. Strictly aerobic and chemo-organotrophic. Catalase-positive. Nitrate is reduced. Ubiquinone is predominantly Q-10. Satu- rated 18:1 is the dominant fatty acid and 16:0 is present as a minor component. Major 2-hydroxy fatty acid is 2-OH 14:0. GSLs are present. Spermidine is the major polyamine component. The DNA G + C content is $62-67 \mathrm{~mol} \%$. Characteristic $16 \mathrm{~S}$ rRNA signatures are present at positions 52:359 (U:A), $134(\mathrm{G}), 593$ (U), 987:1218 (A:U) and 990:1215 (U:G). Habitat: soil, roots of rose tree, clinical specimens, stocked distilled water, coastal plain sediments and fluidizedbed reactor. The type species is Novosphingobium capsulatum (Yabuuchi et al. 1990) Takeuchi et al. 2001.

\section{Description of Novosphingobium aromaticivorum comb. nov.}

With the addition of the details listed in the genus description above, the description is the same as that given by Balkwill et al. (1997).

\section{Description of Novosphingobium capsulatum comb. nov.}

With the addition of the details listed in the genus description above, the description is the same as that given by Yabuuchi et al. (1990).

\section{Description of Novosphingobium roseae comb. nov.}

With the addition of the details listed in the genus description above, the description is the same as that given by Takeuchi et al. (1995).

\section{Description of Novosphingobium stygiae comb. nov.}

With the addition of the details listed in the genus description above, the description is the same as that given by Balkwill et al. (1997).

\section{Description of Novosphingobium subarcticum comb. nov.}

With the addition of the details listed in the genus description above, the description is the same as that given by Nohynek et al. (1996).

\section{Description of Novosphingobium subterraneae comb. nov.}

With the addition of the details listed in the genus description above, the description is the same as that given by Balkwill et al. (1997).

\section{Description of Sphingopyxis gen. nov.}

Sphingopyxis (Sphin.go.pyx'is. Gr. adj. sphingos of sphinx; Gr. n. pyxis jewel box; N.L. fem. n. Sphingopyxis jewel box of sphingolipid-containing life).

Cells are Gram-positive, non-sporulating rods measuring $0.3-0.5 \times 0.9 \mu \mathrm{m}$. Motile or non-motile. 
Colonies are yellow or whitish-brown. Strictly aerobic and chemo-organotrophic. Catalase-positive. Nitrate is not reduced. Ubiquinone is predominantly Q-10. Major fatty acids are 18:1, saturated 16:0 and 16:1. Major 2-hydroxy fatty acids are 2-OH 14:0, 2-OH 15:0 and/or 2-OH 16:0. GSLs are present. Spermidine is the major polyamine component. The DNA G+C content is $63.0-65.0 \mathrm{~mol} \%$. Characteristic $16 \mathrm{~S}$ rRNA signatures are present at positions $52: 359(\mathrm{C}: \mathrm{G}), 134$ $(\mathrm{G}), 593(\mathrm{U}), 987: 1218(\mathrm{G}: \mathrm{C})$ and 990:1215 (U:G). Habitat: soil and underground water. The type species is Sphingopyxis macrogoltabida (Takeuchi et al. 1993) Takeuchi et al. 2001.

\section{Description of Sphingopyxis macrogoltabida comb. nov.}

With the addition of the details listed in the genus description above, the description is the same as that given by Takeuchi et al. (1993).

\section{Description of Sphingopyxis terrae comb. nov.}

With the addition of the details listed in the genus description above, the description is the same as that given by Takeuchi et al. (1993).

\section{ACKNOWLEDGEMENTS}

We are grateful to Dr Kazunori Hatano, Institute for Fermentation, Osaka, for valuable suggestions and discussions. We thank Ms Yayoi Yamaguchi for her help with the fatty acid analysis. A part of this study (by A.H.) was supported by a Grand-in-Aid from the Ministry of Health and Welfare, Japan (Research in Environmental Health, H11 Seikatsu-015).

\section{REFERENCES}

Balkwill, D. L., Drake, G. R., Reeves, R. H. \& 7 other authors (1997). Taxonomic study of aromatic-degrading bacteria from deep-terrestrial-subsurface sediments and description of Sphingomonas aromaticivorans sp. nov., Sphingomonas subterranea sp. nov., and Sphingomonas stygia sp. nov. Int J Syst Bacteriol 47, 191-201.

Brosius, J., Palmer, J. L., Kennedy, J. P. \& Noller, H. F. (1978). Complete nucleotide sequence of a $16 \mathrm{~S}$ ribosomal RNA gene from Escherichia coli. Proc Natl Acad Sci U S A 75, 4801-4805.

Denner, E. B. M., Kämpfer, P., Busse, H.-J. \& Moore, E. R. B. (1999). Reclassification of Pseudomonas echinoides Heumann $1962,343^{\mathrm{AL}}$, in the genus Sphingomonas as Sphingomonas echinoides comb. nov. Int J Syst Bacteriol 49, 1103-1109.

Felsenstein, J. (1985). Confidence limits on phylogenies: an approach using the bootstrap. Evolution 39, 783-791.

Fuerst, J. A., Hawkins, J. A., Holmes, A., Sly, L. I., Moore, C. J. \& Stackebrandt, E. (1993). Porphyrobacter neustonensis gen. nov., sp. nov., an aerobic bacteriochlorophyll-synthesizing budding bacterium from fresh water. Int J Syst Bacteriol 43, 125-134.

Hamana, K. \& Takeuchi, M. (1998). Polyamine profiles as chemotaxonomic marker within alpha, beta, gamma, delta, and epsilon subclasses of class Proteobacteria: distribution of 2- hydroxyputrescine and homospermidine. Microbiol Cult Coll 14, 1-14.

Hamana, K., Sakane, T. \& Yokota, A. (1994). A polyamine analysis of the genera Aquaspirillum, Magnetospirillum, Oceanospirillum and Spirillum. J Gen Appl Microbiol 40, 75-82.

Hiraishi, A., Nagashima, K. V. P., Matsuura, K., Shimada, K., Takaichi, S., Wakao, N. \& Katayama, Y. (1998). Phylogeny and photosynthetic features of Thiobacillus acidophilus and related acidophilic bacteria: its transfer to the genus Acidiphilium as Acidiphilium acidophilum comb. nov. Int J Syst Bacteriol 48, 1389-1398.

Hiraishi, A., Kuraishi, H. \& Kawahara, K. (2000). Emendation of the description of Blastomonas natatoria (Sly 1985) Sly and Cahill 1997 as an aerobic photosynthetic bacterium and reclassification of Erythromonas ursincola Yurkov et al. 1997 as Blastomonas ursincola comb. nov. Int J Syst Evol Microbiol 50, 1113-1118.

Kämpfer, P., Denner, E. B. M., Meyer, S., Moore, E. R. B. \& Busse, H.-J. (1997). Classification of "Pseudomonas azotocolligans" Anderson 1955, 132, in the genus Sphingomonas as Sphingomonas trueperi sp. nov. Int J Syst Bacteriol 47, 577-583.

Kawahara, K., Seydel, U., Matsuura, M., Danbara, H., Rietschel, E. T. \& Zähringer, U. (1991). Chemical structure of glycosphingolipids isolated from Sphingomonas paucimobilis. FEBS Lett 292, 107-110.

Kikuchi, Y., Ozawa, M., Tohmoto, K., Kanamaru, T. \& Sakane, T. (1998). Corrosion behavior of copper and its welds by bacteria in underground water. Trans Joining Welding Res Inst Osaka Univ 27, 53-62.

Kimura, M. (1980). A simple method for estimating evolutionary rates of base substitutions through comparative studies of nucleotide sequences. J Mol Evol 16, 111-120.

Lapage, S. P., Sneath, P. H. A., Lessel, E. F., Skerman, V. B. D., Seeliger, H. P. R. \& Clark, W. A. (editors). (1992). International Code of Nomenclature of Bacteria (1990 Revision). Washington, DC: American Society for Microbiology.

Nohynek, L. J., Suhonen, E. L., Nurmiaho-Lassila, E.-L., Hantula, J. \& Salkinoja-Salonen, M. (1995). Description of four pentachlorophenol-degrading bacterial strains as Sphingomonas chlorophenolica sp. nov. Syst Appl Microbiol 18, 527-538.

Nohynek, L. J., Nurmiaho-Lassila, E.-L., Suhonen, E. L., Busse, H.-J., Mohammadi, M., Hantula, J., Rainey, F. \& SalkinojaSalonen, M. S. (1996). Description of chlorophenol-degrading Pseudomonas sp. strains $\mathrm{KF} 1^{\mathrm{T}}, \mathrm{KF} 3$, and $\mathrm{NKF} 1$ as a new species of the genus Sphingomonas, Sphingomonas subarctica sp. nov. Int J Syst Bacteriol 46, 1042-1055.

Oyaizu-Masuchi, Y. \& Komagata, K. (1988). Isolation of freeliving nitrogen-fixing bacteria from the rhizosphere of rice. $J$ Gen Appl Microbiol 34, 127-164.

Saitou, N. \& Nei, M. (1987). The neighbor-joining method: a new method for reconstructing phylogenetic trees. Mol Biol Evol 4, 406-425.

Segers, P., Vancanneyt, M., Pot, B., Torck, U., Hoste, B., Dewettinck, D., Falsen, E., Kersters, K. \& De Vos, P. (1994). Classification of Pseudomonas diminuta Leifson and Hugh 1954 and Pseudomonas vesicularis Büsing, Döll, and Freytag 1953 in Brevundimonas gen. nov. as Brevundimonas diminuta comb. nov. and Brevundimonas vesicularis comb. nov., respectively. Int $J$ Syst Bacteriol 44, 499-510.

Shiba, T. \& Simidu, U. (1982). Erythrobacter longus gen. nov., sp. nov., an aerobic bacterium which contains bacteriochlorophyll a. Int J Syst Bacteriol 32, 211-217. 
Sly, L. I. \& Cahill, M. M. (1997). Transfer of Blastobacter natatorius (Sly 1985) to the genus Blastomonas gen. nov. as Blastomonas natatoria comb. nov. Int $J$ Syst Bacteriol 47, 566-568.

Stackebrandt, E., Murray, R. G. E. \& Trüper, H. G. (1988). Proteobacteria classis nov., a name for the phylogenetic taxon that includes the "purple bacteria and their relatives". Int J Syst Bacteriol 38, 321-325.

Swofford, D. L. (2000). PAUP*. Phylogenetic analysis using parsimony (*and other methods). Version 4.0b4a. Sunderland, MA: Sinauer Associates.

Takeuchi, M., Kawai, F., Shimada, Y. \& Yokota, A. (1993). Taxonomic study of polyethylene glycol-utilizing bacteria: emended description of the genus Sphingomonas and new descriptions of Sphingomonas macrogoltabidus sp. nov., Sphingomonas sanguis sp. nov., and Sphingomonas terrae sp. nov. Syst Appl Microbiol 16, 227-238.

Takeuchi, M., Sawada, H., Oyaizu, H. \& Yokota, A. (1994). Phylogenetic evidence for Sphingomonas and Rhizomonas as nonphotosynthetic members of the alpha- 4 subclass of the Proteobacteria. Int J Syst Bacteriol 44, 308-314.

Takeuchi, M., Sakane, T., Yanagi, M., Yamasato, K., Hamana, K. \& Yokota, A. (1995). Taxonomic study of bacteria isolated from plants: proposal of Sphingomonas rosa sp. nov., Sphingomonas pruni sp. nov., Sphingomonas asaccharolytica sp. nov., and Sphingomonas mali sp. nov. Int J Syst Bacteriol 45, 334-341.

Thompson, J. D., Higgins, D. G. \& Gibson, T. J. (1994). CLUSTAL $\mathrm{W}$ : improving the sensitivity of progressive multiple sequence alignment through sequence weighting, position-specific gap penalties and weight matrix choice. Nucleic Acids Res 22, 4673-4680.

Van Bruggen, A. H. C., Jochimsen, K. N. \& Brown, P. R. (1990). Rhizomonas suberifaciens gen. nov., sp. nov., the causal agent of corky root of lettuce. Int J Syst Bacteriol 40, 175-188.

Van Bruggen, A. H. C., Jochimsen, K. N., Steinberger, E. M., Segers, P. \& Gillis, M. (1993). Classification of Rhizomonas suberifaciens, an unnamed Rhizomonas species, and Sphingomonas spp. in rRNA superfamily IV. Int $J$ Syst Bacteriol 43, $1-7$.
Yabuuchi, E., Yano, I., Oyaizu, H., Hashimoto, Y., Ezaki, T. \& Yamamoto, H. (1990). Proposals of Sphingomonas paucimobilis gen. nov. and comb. nov., Sphingomonas parapaucimobilis sp. nov., Sphingomonas yanoikuyae sp. nov., Sphingomonas adhaesiva sp. nov., Sphingomonas capsulata comb. nov., and two genospecies of the genus Sphingomonas. Microbiol Immunol 34, 99-119.

Yabuuchi, E., Kosako, Y., Naka, T., Suzuki, S. \& Yano, I. (1999). Proposal of Sphingomonas suberifaciens (van Brüggen, Jochimsen and Brown 1990) comb. nov., Sphingomonas natatoria (Sly 1985) comb. nov., Sphingomonas ursincola (Yurkov et al. 1997) comb. nov., and emendation of the genus Sphingomonas. Microbiol Immunol 43, 339-349.

Yokota, A., Takeuchi, M. \& Weiss, N. (1993). Proposal of two new species in the genus Microbacterium: Microbacterium dextranolyticum sp. nov. and Microbacterium aurum sp. nov. Int $J$ Syst Bacteriol 43, 549-554.

Yrjälä, K., Suomalainen, S., Suhonen, E. L., Kilpi, S., Paulin, L. \& Romantschuk, M. (1998). Characterization and reclassification of an aromatic- and chloroaromatic-degrading Pseudomonas sp., strain HV3, as Sphingomonas sp. HV3. Int J Syst Bacteriol 48, 1057-1062.

Yurkov, V., Stackebrandt, E., Holmes, A. \& 7 other authors (1994). Phylogenetic positions of novel aerobic, bacteriochlorophyll $a$-containing bacteria and description of Roseococcus thiosulfatophilus gen. nov., sp. nov., Erythromicrobium ramosum gen. nov., sp. nov., and Erythrobacter litoralis sp. nov. Int J Syst Bacteriol 44, 427-434.

Yurkov, V., Stackebrandt, E., Buss, O., Vermeglio, A., Gorlenko, V. \& Beatty, J. T. (1997). Reorganization of the genus Erythromicrobium: description of "Erythromicrobium sibiricum" as Sandaracinobacter sibiricus gen. nov., sp. nov., and of " Erythromicrobium ursincola" as Erythromonas ursincola gen. nov., sp. nov. Int J Syst Bacteriol 47, 1172-1178.

Zipper, C., Nickel, K., Angst, W. \& Kohler, H. (1996). Complete microbial degradation of both enantiomers of the chiral herbicide mecoprop [(RS)-2-(4-chloro-2-methylphenoxy) propionic acid] in an enantioselective manner by Sphingomonas herbicidovorans sp. nov. Appl Environ Microbiol 62, 4318-4322. 